\title{
Hydrodynamics of Leidenfrost droplets in one-component fluids
}

\author{
Xinpeng $\mathrm{Xu}^{1}$ and Tiezheng Qian ${ }^{2, *}$ \\ ${ }^{1}$ Department of Mathematics, Hong Kong University of Science and Technology, Clear Water Bay, Kowloon, Hong Kong \\ ${ }^{2}$ Department of Mathematics and KAUST-HKUST Micro/Nanofluidics Joint Laboratory, Hong Kong University of Science and Technology, \\ Clear Water Bay, Kowloon, Hong Kong
}

(Received 4 December 2012; published 24 April 2013)

\begin{abstract}
Using the dynamic van der Waals theory [Phys. Rev. E 75, 036304 (2007)], we numerically investigate the hydrodynamics of Leidenfrost droplets under gravity in two dimensions. Some recent theoretical predictions and experimental observations are confirmed in our simulations. A Leidenfrost droplet larger than a critical size is shown to be unstable and break up into smaller droplets due to the Rayleigh-Taylor instability of the bottom surface of the droplet. Our simulations demonstrate that an evaporating Leidenfrost droplet changes continuously from a puddle to a circular droplet, with the droplet shape controlled by its size in comparison with a few characteristic length scales. The geometry of the vapor layer under the droplet is found to mainly depend on the droplet size and is nearly independent of the substrate temperature, as reported in a recent experimental study [Phys. Rev. Lett. 109, 074301 (2012)]. Finally, our simulations demonstrate that a Leidenfrost droplet smaller than a characteristic size takes off from the hot substrate because the levitating force due to evaporation can no longer be balanced by the weight of the droplet, as observed in a recent experimental study [Phys. Rev. Lett. 109, 034501 (2012)].
\end{abstract}

DOI: 10.1103/PhysRevE.87.043013

PACS number(s): 47.55.D-, 68.08.-p, 44.35.+c, 47.61.-k

\section{INTRODUCTION}

Boiling and evaporation of liquids are commonly observed in our daily life $[1,2]$. Consider a liquid droplet deposited on a hot solid surface. If the solid temperature is a bit above the boiling point of the liquid, then the droplet will boil and evaporate rapidly. However, if the solid temperature is much higher than the boiling point, then a vapor layer is formed between the droplet and the solid [1,3]. A droplet levitating over a vapor layer on a hot solid surface is called a Leidenfrost droplet, named after the German medical doctor Johann Gottlob Leidenfrost, who first reported the phenomena in 1756 [4]. In the presence of the vapor layer, the droplet avoids a direct contact with the solid surface. The consequences are twofold: First, the vapor layer acts as a thermally insulating layer due to its small heat conductivity. This reduces the rate of heat transfer into the droplet and prevents the bubble nucleation so the droplet does not boil but evaporates slowly [3,5]. Therefore, the droplet lifetime is greatly extended. Second, the Leidenfrost droplet acquires high mobility on the vapor layer $[3,6,7]$.

Over the years, the Leidenfrost phenomena have attracted continuous pure and applied research efforts. This may be partly attributed to a variety of technological applications involving liquid droplets evaporating on hot surfaces $[1,8]$. These include quenching of metals, quick-response steam generators, spray drying, spray cooling of nuclear reactor cores, and film cooling of rocket nozzles. Recently, the rich behaviors of the Leidenfrost droplets have become better understood with the help of high speed and ultra-high-speed video imaging, surface coating, and micromachining [3,5-7,9-17]. Below are a few key aspects of the Leidenfrost phenomena.

(i) Determination of the Leidenfrost temperature $T_{L}[1,18]$. The Leidenfrost temperature is defined as the temperature

*Corresponding author. E-mail address: maqian@ust.hk of the hot solid surface at which the lifetime of the liquid droplet attains a maximum. This is the temperature above which a stable thermally insulating vapor layer can be fully developed to prevent the liquid from touching the solid. The determination of $T_{L}$ involves both thermodynamics and hydrodynamics: the nucleation of vapor bubbles due to thermodynamic fluctuations, the growth of vapor bubbles on the surface due to surface heating, and the pinch-off of bubbles from the surface due to gravity. Together, they determine whether the formation and growth of randomly distributed vapor bubbles on the surface can generate a continuous stable vapor layer beneath the liquid droplet.

(ii) The Leidenfrost droplets have different sizes $[5,15,19$, 20]. A droplet larger than a critical (maximum) size $\left(R_{\max } \approx\right.$ $4 R_{c}$ with $R_{c}$ being the capillary length) becomes unstable due to the Rayleigh-Taylor instability of the bottom surface of the droplet $[5,19]$. For droplets smaller than $R_{\max }$, three different regimes can be identified as follows for decreasing droplet radius. (a) Puddles (disk- or pancake-shaped droplets) of radius of the order of magnitude of $R_{c}$. In this regime, as evaporation proceeds, the droplet shrinks and sinks with the vapor layer getting thinner. The droplet thickness $d$, however, remains nearly constant $\left(\approx 2 R_{c}\right)$ [5]. (b) Quasispherical droplets of radius of the order of magnitude of $R_{i}\left(\ll R_{c}\right)$, a characteristic length scale introduced by Celestini et al. [15]. In this regime, as evaporation proceeds, the droplet shrinks and rises with the vapor layer getting thicker. (c) Spherical droplets of radius much smaller than $R_{i}$. Similar to the quasispherical droplets, as evaporation proceeds, they shrink and rise.

(iii) Spatial and temporal evolution of the vapor layer [16]. Burton et al. [16] accurately measured the radius, curvature, and height of the vapor layer under the Leidenfrost (water) droplets at different temperatures. They found that the geometry of the vapor layer depends primarily on the droplet size and not on the substrate temperature.

(iv) Take-off of small Leidenfrost droplets [15,20]. Celestini et al. [15] predicted and observed that, below a critical 
size $R_{l}$, the spherical Leidenfrost droplets take off from the hot solid surfaces.

In short, the dynamics of the Leidenfrost droplets is characterized by the coupling of mass flow, free interface motion, heat conduction, and phase change (evaporation) $[1,3,20]$. This makes it a complicated problem exhibiting rich behaviors. Therefore, modeling and simulation efforts made for its understanding are not only of theoretical interest but also necessitated by the rapid advances in experimental studies.

Recently, the dynamic van der Waals theory (DVDWT) has been presented for one-component van der Waals fluids. This theory is a phase-field model capable of describing the two-phase hydrodynamics involving the liquid-vapor phase transition in inhomogeneous temperature field [21,22]. In addition, the boundary conditions for two-phase hydrodynamics on solid surfaces have been derived by considering the dissipative processes at the fluid-solid interface [23-26]. In the phase-field modeling, the density field (and, hence, the liquid-vapor interfacial profile), velocity field, and temperature field are governed by a single set of partial differential equations. In comparison with other modeling methods for multiphase flows involving the liquid-vapor phase transition [20,27-31], the phase-field modeling does not need interfacial boundary conditions at the liquid-vapor interface [22] and, hence, the rate of evaporation is not a prerequisite input but a computational output. Given these advantages, the DVDWT has been used to study pool boiling [22,32], droplet evaporation on heated surfaces without gravity [33], droplet spreading on heated or cooled substrates [34], droplet motion induced by wettability and thermal gradients [25,26], and so on. It is interesting to note that Teshigawara et al. [33] applied the DVDWT in an axisymmetric geometry and observed the Leidenfrost phenomena in van der Waals fluids without gravity. Nevertheless, for Leidenfrost droplets under gravity, no phase-field approach to droplet dynamics has been reported.

The purpose of the present work is to employ the DVDWT to investigate the Leidenfrost droplet dynamics in van der Waals fluids under gravity. We numerically implement the DVDWT in a two-dimensional geometry by using an explicit finite difference scheme [23-26]. We then study the evaporation of a Leidenfrost droplet surrounded by its own vapor. We focus on the evolution of the droplet shape and the geometry of the vapor layer. Our numerical results demonstrate different scaling regimes predicted by recent scaling analyses $[15,16,20]$. The take-off of small circular droplets, which invalidates the commonly used lubrication approximation [15], is also numerically observed. The liquid-vapor coexistence temperature and pressure are initially set to be close to the critical point, at $0.875 T_{c}$ and $0.584 p_{c}$, respectively, with $T_{c}$ being the critical temperature and $p_{c}$ the critical pressure. As a consequence, the liquid-to-vapor density ratio is only about 5 and the small mean free path in the vapor justifies the phase-field approach [34]. In order to make the different scaling regimes numerically accessible and observable, we make use of artificially large gravitational accelerations [22]. Regardless of the above assumptions made for the numerical computation, our results are able to confirm and reproduce some recent theoretical predictions and experimental observations $[3,5,15,16,19,20]$ for Leidenfrost droplets exposed in the air at room temperatures and room pressures. It is our hope that alternative numerical methods can be combined with our model to produce more realistic simulations in more complicated geometries [35].

The paper is organized as follows. Section II is a review of the dynamic van der Waals theory for one-component liquid-vapor systems on solid substrates. A description of the numerical implementation is also given. In Sec. III, numerical results are presented and discussed. Some important theoretical predictions and experimental observations are numerically observed in a series of two-dimensional simulations. The paper is concluded in Sec. IV with a few remarks.

\section{THEORETICAL MODELING AND NUMERICAL IMPLEMENTATION}

This section is a brief review of the dynamic van der Waals theory. The numerical implementation is also described.

\section{A. Dynamic van der Waals theory}

Consider a one-component liquid-vapor system on a solid surface, which is assumed to be flat, rigid, homogeneous, and of high heat conductivity [24-26]. The dynamic van der Waals theory (DVDWT) [21,22] for one-component fluids can be outlined as follows.

In the van der Waals theory for homogeneous onecomponent monatomic fluids [22,36], the fluids are characterized by the molecular volume $v_{0}=a_{\mathrm{vdw}}^{3}$ (with $a_{\mathrm{vdw}} \equiv v_{0}^{1 / 3}$ being the order of magnitude of the molecular diameter) and the attractive interaction energy $\varepsilon$. The Helmholtz free energy density is given by $f(n, T)=n k_{B} T \ln \left[\lambda_{\mathrm{th}}^{3} n /\left(1-v_{0} n\right)\right]-$ $n k_{B} T-\varepsilon v_{0} n^{2}$, from which the entropy per molecule $s$, the internal energy density $e$, and the pressure $p$ can be obtained

$$
\begin{gathered}
s(n, T)=k_{B} \ln \left[\left(k_{B} T / \varepsilon\right)^{3 / 2}\left(1 / n v_{0}-1\right)\right]+\text { const, } \\
e(n, T)=3 n k_{B} T / 2-\varepsilon v_{0} n^{2} \\
p(n, T)=n k_{B} T /\left(1-v_{0} n\right)-\varepsilon v_{0} n^{2} .
\end{gathered}
$$

Here $n$ is the number density, $T$ is the temperature, $k_{B}$ is the Boltzmann constant, and $\lambda_{\text {th }}=\hbar\left(2 \pi / m k_{B} T\right)^{1 / 2}$ is the thermal de Broglie wavelength with $m$ and $\hbar$ being the molecular mass and the Planck constant. In this mean-field theory, the critical temperature, critical pressure, and critical density are given by $T_{c}=8 \varepsilon / 27 k_{B}, p_{c}=\varepsilon / 27 v_{0}$, and $n_{c}=1 / 3 v_{0}$, respectively. At a given temperature $T<T_{c}$, liquid and vapor phases can coexist at a coexistence pressure $p_{\mathrm{cx}}$, with the temperature $T$ referred to as the coexistence temperature and denoted by $T_{\mathrm{cx}}$. For example, at the coexistence temperature $T_{\mathrm{cx}}=0.875 T_{c}$, the coexistence pressure is $p_{\mathrm{cx}} \approx 0.584 p_{c}$, and the liquid and vapor densities are $n_{l} \approx 0.58 / v_{0}$ and $n_{v} \approx 0.122 / v_{0}$, respectively. (See the appendix for the experimental data for water and nitrogen at a coexistence temperature $T_{\mathrm{cx}} \approx 0.9 T_{c}$.)

To describe the inhomogeneous van der Waals fluids, gradient contributions must be included. As in most of the phase-field models for one-component liquid-vapor systems [37,38], the local number density $n=n(\mathbf{r}, t)$ is used as the order parameter, which takes distinct values in the liquid and 
vapor phases and shows a fast variation across the liquid-vapor interface. In nonisothermal situations, the conventional use of free-energy functional is inapplicable. According to Onuki $[21,22]$, we should instead start with an entropy functional $S_{b}=\int d \mathbf{r} \hat{S}$ by including a gradient contribution. The local entropy density $\hat{S}=\hat{S}(\mathbf{r}, t)$ consists of a regular term for homogeneous fluids and a gradient term arising from the density inhomogeneity:

$$
\hat{S}=n s(n, e)-\frac{C}{2}|\nabla n|^{2} .
$$

Note that the entropy per molecule $s(n, e)$ as a function of the number density $n$ and the internal energy density $e$ can be derived from Eqs. (1) and (2) by eliminating $T$. The coefficient $C$ is a positive constant, indicating a decrease of entropy due to the density inhomogeneity. Moreover, the internal energy functional $E_{b}$ is assumed to be the space integral of $e$, i.e., $E_{b}=\int d \mathbf{r} e$. More generally [21,22], the coefficient $C$ may depend on $n$, and the internal energy functional $E_{b}$ may also contain a gradient contribution. In the present work, we include a constant $C$ in $\hat{S}$ and neglect the gradient contribution to $E_{b}$. This will not affect the essential features of our results [21,22].

The boundary effects of the solid substrate on the fluids can be included by introducing the surface entropy $S_{s}=$ $\int d A \sigma_{s}(n)$ and the surface energy $E_{s}=\int d A e_{s}(n)$. Here $\int d A$ denotes the surface integral on the solid surface, and areal densities $\sigma_{s}$ and $e_{s}$ are assumed to only depend on the boundary value of fluid density at the solid surface.

Maximizing the total entropy $S_{\mathrm{tot}}=S_{b}+S_{s}$ subject to fixed particle number $N=\int d \mathbf{r} n$ and fixed total internal energy $E_{\text {tot }}=E_{b}+E_{s}$ leads to the equilibrium conditions: (i) $1 / T \equiv$ $\left(\delta S_{b} / \delta e\right)_{n}=n(\partial s / \partial e)_{n}=\mathrm{const}, \quad$ (ii) $\hat{\mu} \equiv-T\left(\delta S_{b} / \delta n\right)_{e}=$ $\mu-C T \nabla^{2} n=$ const, and (iii)

$$
L \equiv C T \nabla_{\gamma} n+\left(\partial f_{s} / \partial n\right)_{T}=0,
$$

at the solid surface with $\nabla_{\gamma} \equiv \hat{\gamma} \cdot \nabla$ and $\hat{\gamma}$ denoting the outward unit vector normal to the surface. Here $\mu \equiv$ $-T[\partial(n s) / \partial n]_{e}$ is the chemical potential for homogeneous fluids and $f_{s}(n, T) \equiv e_{s}(n)-T \sigma_{s}(n)$ is the areal density of surface free energy.

Due to the presence of the gradient entropy in Eq. (4), there arises a new length scale $\ell$ defined by $[21,22]$

$$
\ell \equiv\left(C / 2 k_{B} v_{0}\right)^{1 / 2}
$$

besides the molecular diameter $a_{\mathrm{vdw}} \equiv v_{0}^{1 / 3}$. For real simple fluids, the ratio $\ell / a_{\mathrm{vdw}}$ is of order unity (see the appendix for the estimations of $\ell$ and $a_{\mathrm{vdw}}$ using the experimental data for water and nitrogen). In the present phase-field approach, we treat $\ell$ as an adjustable parameter. The liquid-vapor interfacial thickness is of the order of magnitude of $\ell$ far from the critical point and varies as $\ell\left(1-T / T_{c}\right)^{-1 / 2}$ close to the critical point [34]. The surface tension $\gamma$ is of the order of magnitude of [34]

$$
\gamma \sim k_{B} T\left(1-T / T_{c}\right)^{3 / 2} \ell / v_{0} .
$$

Now we present the hydrodynamic equations which can be derived from the principle of positive entropy production in nonequilibrium thermodynamics [36,39]. The mass density $\rho \equiv m n$, momentum density $\rho \mathbf{v}$, and total energy density
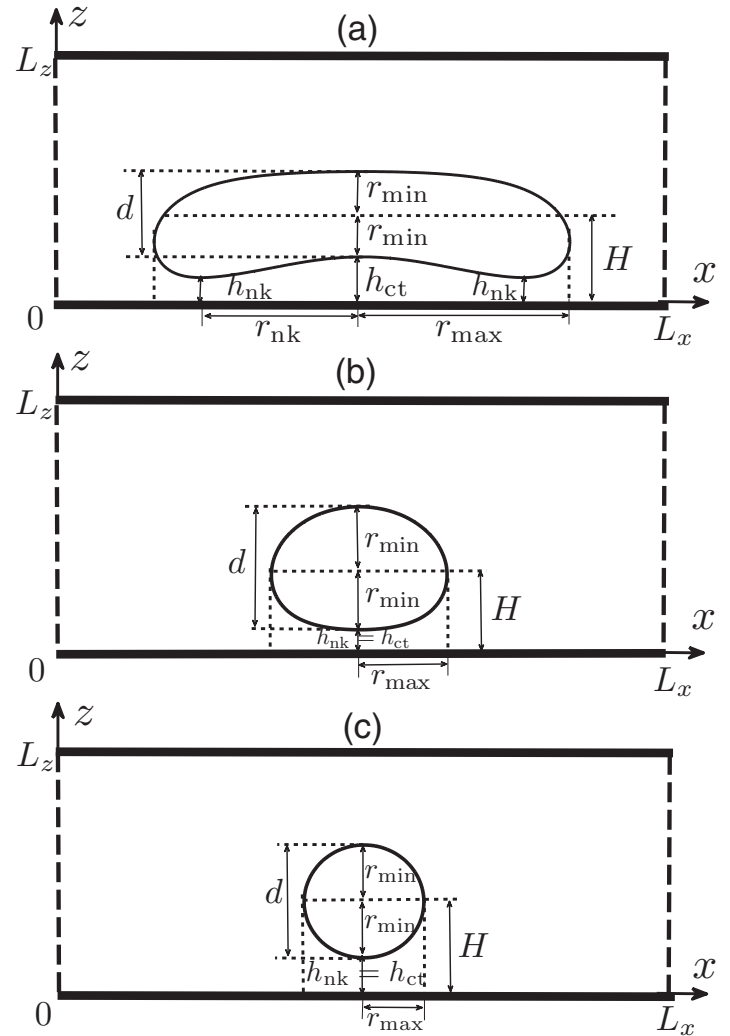

FIG. 1. Schematic illustrations for Leidenfrost droplets of different shapes. (a) A puddle. (b) A quasicircular droplet. (c) A circular droplet. A few lengths characterizing the droplet shape and the geometry of the vapor layer are defined here. The droplet thickness is measured by $d$, which is related to the radius $r_{\min }$ via $r_{\min }=d / 2$. The other two radii are $r_{\max }$ and $r_{\mathrm{nk}}$, which are the maximum radius and the neck radius of the droplet, respectively. As to the geometry of the vapor layer, its thickness is measured by $h_{\mathrm{ct}}$ at the center and $h_{\mathrm{nk}}$ at the neck, respectively. The height of the droplet is measured by $H \equiv h_{\mathrm{ct}}+r_{\min }$. In addition, an effective radius of the droplet $r_{\mathrm{eff}}$ can be defined via $\pi r_{\text {eff }}^{2}=$ the instantaneous droplet area.

$e_{T} \equiv e+\rho \mathbf{v}^{2} / 2$ satisfy the balance equations

$$
\begin{gathered}
\frac{\partial n}{\partial t}+\nabla \cdot(n \mathbf{v})=0, \\
\frac{\partial}{\partial t}(\rho \mathbf{v})+\nabla \cdot(\rho \mathbf{v v})=\nabla \cdot \overleftrightarrow{\mathbf{M}}-\rho g \mathbf{e}_{z}, \\
\frac{\partial e_{T}}{\partial t}+\nabla \cdot\left(e_{T} \mathbf{v}\right)=\nabla \cdot(\stackrel{\leftrightarrow}{\mathbf{M}} \cdot \mathbf{v})-\nabla \cdot \mathbf{q}-\rho g v_{z},
\end{gathered}
$$

where $g$ is the gravitational acceleration, $\mathbf{e}_{z}$ is the upward unit vector along the $z$ axis (as shown in Fig. 1), and $v_{z} \equiv \mathbf{v} \cdot \mathbf{e}_{z}$ is the $z$ component of the fluid velocity $\mathbf{v}$. The total stress tensor $\overleftrightarrow{\mathbf{M}}=-\overleftrightarrow{\Pi}+\overleftrightarrow{\boldsymbol{\sigma}}$ consists of the irreversible viscous part $[36,39]$ $\overleftrightarrow{\boldsymbol{\sigma}}=\eta\left(\nabla \mathbf{v}+\nabla \mathbf{v}^{T}\right)+(\zeta-2 \eta / 3) \overleftrightarrow{\mathbf{I}} \nabla \cdot \mathbf{v}$ and the reversible part

$$
-\stackrel{\Pi}{\Pi}=-C T \nabla n \nabla n-\hat{p} \overleftrightarrow{\mathbf{I}},
$$

in which the anisotropic part $-C T \nabla n \nabla n$ results in the liquidvapor interfacial tension [21,22], and $\hat{p}$ is the generalized pressure given by $\hat{p} \equiv p-C T|\nabla n|^{2} / 2-C T n \nabla^{2} n$ with $p$ given by Eq. (3). The heat flux $\mathbf{q}$ is given by $\mathbf{q}=-\lambda \nabla T$ $[36,39,40]$. Here the transport coefficients $\eta, \zeta$, and $\lambda$ denote 
the shear viscosity, bulk viscosity, and heat conductivity, respectively.

Assuming local equilibrium (for slow hydrodynamics) and using equations (4), (8)-(10), we can obtain the balance equation for the entropy density $\hat{S}[21,22]$

$$
\frac{\partial \hat{S}}{\partial t}+\nabla \cdot(\hat{S} \mathbf{v})=-\nabla \cdot \hat{\mathbf{J}}_{f}^{S}+\sigma
$$

in which the density of the rate of entropy production $\sigma \equiv \overleftrightarrow{\boldsymbol{\sigma}}: \nabla \mathbf{v} / T-\mathbf{q} \cdot \nabla T / T^{2}$ must be positive definite according to the second law of thermodynamics and $\hat{\mathbf{J}}_{f}^{S} \equiv$ $[\mathbf{q} / T+C(\partial n / \partial t+\mathbf{v} \cdot \nabla n) \nabla n]$ is the total reversible entropy flux, including the contribution of the number density variation.

To close the above system of partial differential equations, we employ the following boundary conditions at the solid surface [22,24].

(i) For the fluid velocity $\mathbf{v}$, the no-slip boundary condition is applied, i.e., $\mathbf{v}=0$ (in the reference frame moving where the solid substrate is still) [40].

(ii) For the fluid temperature $T$, the Dirichlet boundary condition is applied, i.e., $T=T_{w}$ with the substrate temperature $T_{w}$ being a given constant [22,34].

(iii) For the number density $n$, the equilibrium condition in Eq. (5) is applied, i.e., $L \equiv M \nabla_{\gamma} n+\left(\partial f_{s} / \partial n\right)_{T}=0$. This assumes fast equilibration of the density at the solid boundary [22].

It is worth pointing out that recent molecular dynamics simulations have shown that velocity slip (tangential to the solid surface) [41-43] and temperature jump (due to the Kapitza resistance) [44-46] can both occur at the fluid-solid interface, especially when the solid surface is hydrophobic. These phenomena have been discussed and modeled by a more general set of boundary conditions in our previous work [24-26]. The simplified boundary conditions used here are justified by the fact that the Leidenfrost droplets levitate on a vapor cushion at the solid substrate such that there is no direct contact between the liquid and the solid and, hence, no contact line is involved in the droplet dynamics. A detailed modeling of the physical processes at the fluid-solid interface therefore becomes nonessential [3,20,47]. We would like to emphasize that a detailed modeling of the interfacial processes does become essential in describing the formation of the vapor layer and in determining the Leidenfrost temperature $[1,18]$.

\section{B. Simulation details}

The hydrodynamic equations (8), (9), and (12) are integrated in the two-dimensional $x z$ plane, with the liquid-vapor mixture confined in the region $0 \leqslant x \leqslant L_{x}$ and $0 \leqslant z \leqslant L_{z}$ (see Fig. 1). The two fluid-solid interfaces are defined at $z=0$ and $L_{z}$, and periodic boundary condition is applied in the $x$ direction to close the system. The state variables $n, \mathbf{v}$, and $T$ in the fluid are defined in a two-dimensional, unstaggered, uniformly discretized Cartesian mesh [24-26]. The mesh size is $\Delta x=\Delta z=0.5 \ell$, which is fine enough to resolve the liquid-vapor interface (of thickness $\approx 2.5 \ell$ ) in our simulations $[25,26]$. It is important to note that to update the fluid temperature $T$, the entropy equation (12) is integrated instead of the energy equation (10) to avoid artificial parasitic flows [33]. The local relation between $T$ and $\hat{S}$ can be obtained from Eqs. (4) and (1). [Note that the constant in Eq. (1) can be omitted because $\hat{S}$ is determined up to $n \times$ const by Eq. (12) because of the continuity equation (8)]. Limited by our computational capability, $L_{x}$ and $L_{z}$ are of the order of magnitude of $100 \ell$, and the droplets are as large as a few tens of $\ell$. As $\ell \sim 1 \mathrm{~nm}[34,48]$, our systems are very small and the droplets are of the size of a few tens of nanometers. We would like to point out that more realistic simulations at larger length scales can be made possible with the help of adaptive mesh method [49].

For simplicity, we assume that the transport coefficients are of the forms $\eta=\zeta=v m n$ and $\lambda=v k_{B} n$, with linear dependence on the local number density [50]. The kinematic viscosity $v=\eta / \rho$ is, therefore, a constant independent of $n$. Under these assumptions, on the one hand, the transport coefficients are larger in the liquid than in the vapor by a factor of $n_{l} / n_{v}$ ( $\approx 5$ in our simulations). On the other hand, since the isobaric specific heat capacity $C_{p}$ per unit volume is about $k_{B} n$, the thermal diffusivity $D_{T}=\lambda / C_{p}$ is about $v$ and, hence, the Prandtl number $\operatorname{Pr}=v / D_{T}$ is of order unity in both phases. (For a justification of our assumptions, see the appendix for the experimental data for water and nitrogen.) Finally, the surface energy density and surface entropy density are assumed to be of the forms $e_{s}=$ const and $\sigma_{s}=-c_{s}\left(n-n_{c}\right)$, respectively, with $c_{s}$ being a constant independent of $n[25,34]$. The surface free energy density is then given by $f_{s}=$ const $+c_{s} T\left(n-n_{c}\right)$. Widely used in the study of wetting phenomena [34,38,51], this kind of surface free energy function leads to density enrichment or depletion near the solid surface.

In our calculations, the space is measured by $\ell$ and the time is measured by $\tau_{0} \equiv \ell^{2} / \nu$, the viscous relaxation time at the length scale of $\ell$. (Note that $\tau_{0}$ also gives the order of magnitude of the thermal diffusion time at the same length scale because of $\operatorname{Pr} \sim 1$.) The number density is measured by $1 / v_{0}$ and the temperature is measured by the critical temperature $T_{c}$. Dedimensionalizing our equation system [22,25], we obtain three dimensionless parameters: $\mathcal{R} \equiv v^{2} m / \varepsilon \ell^{2}$,

$$
\mathcal{G} \equiv m g \ell / \varepsilon,
$$

which is the dimensionless gravitational acceleration, and $\mathcal{W} \equiv c_{s} / k_{B} \ell$ from the surface free energy, which determines the static contact angle for a droplet in direct contact with the solid [25]. In the present work, we use $\mathcal{R}=0.06[22,33,34]$ (see the appendix for the estimation of $\mathcal{R}$ using the experimental data for water and nitrogen) and $\mathcal{W}=0$, which gives a static contact angle of $90^{\circ}[25,33]$.

An explanation is in order for the use of the dimensionless gravitational acceleration $\mathcal{G}$. From its expression, $\mathcal{G}$ is the gravitational energy of a molecule over the distance $\ell$ measured by the van der Waals energy $\varepsilon$. For $g=9.8 \mathrm{~m} / \mathrm{s}^{2}$ on the surface of the Earth and $\ell \sim a_{\mathrm{vdw}}, \mathcal{G}$ is extremely small ( $\mathcal{G} \sim 10^{-14}$ for water and nitrogen, see the appendix for the estimation of $\mathcal{G}$ ). For the purpose of the present work, it is important to note that $\mathcal{G}$ is directly related to the capillary length defined by $R_{c} \equiv\left(\gamma / \rho_{l} g\right)^{1 / 2}$ with $\rho_{l}=n_{l} m$ being the liquid mass density. Physically, the capillary length represents the length scale over which the gravitational potential energy and the liquid-vapor interfacial energy are comparable. At the 
TABLE I. The characteristic length scales $R_{\max }, R_{c}, R_{i}, R_{l}$, and $R_{\min }$ calculated for different dimensionless gravitational accelerations $\mathcal{G}$ used in Secs. III B and III C.

\begin{tabular}{lcrrrrc}
\hline \hline Section & $\mathcal{G}$ & $R_{\max } / \ell$ & $R_{c} / \ell$ & $R_{i} / \ell$ & $R_{l} / \ell$ & $R_{\min } / \ell$ \\
\hline \multirow{4}{*}{ IIIB } & $1.0 \times 10^{-4}$ & 64 & 16 & 11 & 4 & 5 \\
& $5.0 \times 10^{-5}$ & 92 & 23 & 14 & 5 & 5 \\
& $2.5 \times 10^{-5}$ & 126 & 32 & 20 & 6 & 5 \\
& $1.5 \times 10^{-5}$ & 164 & 41 & 25 & 8 & 5 \\
IIIC & $5.0 \times 10^{-6}$ & 288 & 72 & 42 & 11 & 5 \\
& $1.0 \times 10^{-6}$ & 640 & 160 & 88 & 19 & 5 \\
& $5.0 \times 10^{-7}$ & 904 & 226 & 121 & 24 & 5 \\
\hline \hline
\end{tabular}

coexistence temperature $T_{\mathrm{cx}}=0.875 T_{c}$ with $n_{l} \approx 0.58 / v_{0}$, we can obtain an estimation of $R_{c}$ as

$$
R_{c} \equiv\left(\gamma / \rho_{l} g\right)^{1 / 2} \approx 0.14 \ell / \mathcal{G}^{1 / 2}
$$

using $T_{c}=8 \varepsilon / 27 k_{B}$ and Eqs. (7) and (13). For real simple fluids on the surface of the Earth, the capillary length $R_{c}$ is a macroscopic length scale $\left(R_{c} \gg \ell\right)$. That is, for $\ell \sim 1 \mathrm{~nm}$ and $\mathcal{G} \sim 10^{-14}, R_{c} \sim 1 \mathrm{~mm}$ (see the appendix for the estimation of $R_{c}$ using the experimental data for water and nitrogen).

In the present numerical study, in order to induce appreciable gravitational effect on the nanoscale systems, $R_{c}$ has to be treated as an adjustable microscopic parameter $\left(R_{c} \gtrsim \ell\right)$. This is achieved by using artificially large gravitational accelerations $g$ (or equivalently $\mathcal{G}$ ) [22,52,53]. According to Table I, for $\mathcal{G}$ ranging from $2.5 \times 10^{-7}$ to $1.0 \times 10^{-4}, R_{c}$ ranges from $320 \ell$ to $16 \ell$, thus becoming numerically accessible. This makes it possible to investigate the different scaling regimes exhibited by a Leidenfrost droplet as evaporation proceeds. More details will be presented in Sec. III regarding the proper values of $\mathcal{G}$ for observing different evaporation stages. We would like to point out that the use of artificially large gravitational accelerations is, on the one hand, necessitated by the multiscale nature of the Leidenfrost droplet dynamics and, on the other hand, caused by our limited computational capability.

\section{Preparation of the Leidenfrost droplets}

To produce a Leidenfrost droplet, a two-dimensional semicircular liquid droplet of radius $R_{0}$ is placed on the bottom substrate at $z=0$. It is surrounded by its own vapor and under the influence of an artificially large gravitational acceleration $\mathcal{G}$ (see Table I). The temperatures of the fluid, the top substrate, and the bottom substrate are all set to be $0.875 T_{c}$ in the beginning. The liquid density $n_{l}$ and the vapor density $n_{v}$ take their respective values at liquid-vapor coexistence, given by $n_{l} \approx 0.58 / v_{0}$ and $n_{v} \approx 0.122 / v_{0}$ at the coexistence temperature $T_{c x}=0.875 T_{c}$. Under these conditions, we wait until the droplet reaches an almost equilibrium state [25,34] with a static contact angle of $90^{\circ}$ (because of $\mathcal{W}=0$ adopted in our simulations [25]). When this equilibration process is completed, we set $t=0$ as the origin of the time axis. Figure 2 shows a droplet in equilibrium at $t=0$.

To prepare an initial equilibrium state appropriate for a subsequent dynamic simulation, the following must be noted: (i) The computational domain of size $L_{x} \times L_{z}$ should be large enough to avoid possible boundary effects and maintain (nearly) constant ambient pressure. This makes our simulations quite time-consuming. (ii) For a given gravitational acceleration $\mathcal{G}$, the shape of a Leidenfrost droplet can vary from a puddle to a circle, depending on the initial droplet radius $R_{0}$. (Note that the simulations are in two dimensions.) Therefore, to investigate a particular aspect of the droplet dynamics, an appropriate value of $R_{0}$ should be chosen in the beginning. (iii) The initial droplet radius $R_{0}$ cannot be too large. If the maximum radius $r_{\max }$ of a Leidenfrost droplet (defined in Fig. 1) exceeds the maximum size $R_{\max } \approx 4 R_{c}$, then the droplet becomes unstable and breaks up into smaller droplets (see Fig. 2) [5,19].

The temperature of the bottom substrate $T_{w}$ is suddenly raised at $t=0$. The lifetime of the droplet has a strong dependence on this temperature $[1,18]$. In particular, the lifetime of the droplet attains a maximum at a special substrate temperature, namely the Leidenfrost temperature $T_{L}$. Our simulations have shown that the maximum lifetime is indeed achieved by the generation of a stable vapor layer between the droplet and the bottom substrate of temperature $T_{w}=T_{L}$, as reported in early theoretical and experimental works $[1,18]$. We point out that since thermodynamic fluctuations responsible for bubble nucleation are absent in the present mean-field model, the vapor layer is actually generated via the instability of the superheated liquid near the bottom substrate. In real situations, however, both mechanisms are present $[1,18]$.

In most of the experimental studies of droplet evaporation $[1,18]$, a known volume of liquid is deposited on a hot substrate at a fixed temperature, and the total evaporation time (i.e., the lifetime) of the droplet is measured. This lifetime is recorded for the same liquid volume but different substrate temperatures. Our simulations have been carried out accordingly, with the Leidenfrost temperature determined to be $T_{L} \approx 0.97 T_{c}$.

Finally, we mention the two prominent restrictions of our approach: (i) The size of the simulated system, typically at nanometer or submicrometer scale, is limited by the computational capacity $[22,25,43,54]$ because the mesh size of the discretized phase-field model has to be smaller than the length scale $\ell$ of the liquid-vapor interfacial thickness. The real gravitational effect, however, becomes relevant only at macroscopic length scale $[22,40]$. Therefore, it is necessary to use an artificially large gravitational acceleration to incorporate the gravitational effect into a phase-field model [22,52,53]. (ii) Given the finite liquid-vapor interfacial thickness $(\approx 2.5 \ell$ in our simulations), the size of a well-defined droplet must not be smaller than a minimum value, denoted by $R_{\min }$ and chosen to be $5 \ell$ here (see Table I).

\section{RESULTS AND DISCUSSION}

Now we present and discuss the numerical results obtained for the Leidenfrost hydrodynamics. We first outline a scaling analysis in two dimensions. This is to show that the characteristics of the Leidenfrost droplets are determined by the droplet size in comparison with a few characteristic length scales $[5,15,16,20]$. We then numerically investigate the evolution of the droplet shape $[5,15,19,20]$ and that of 


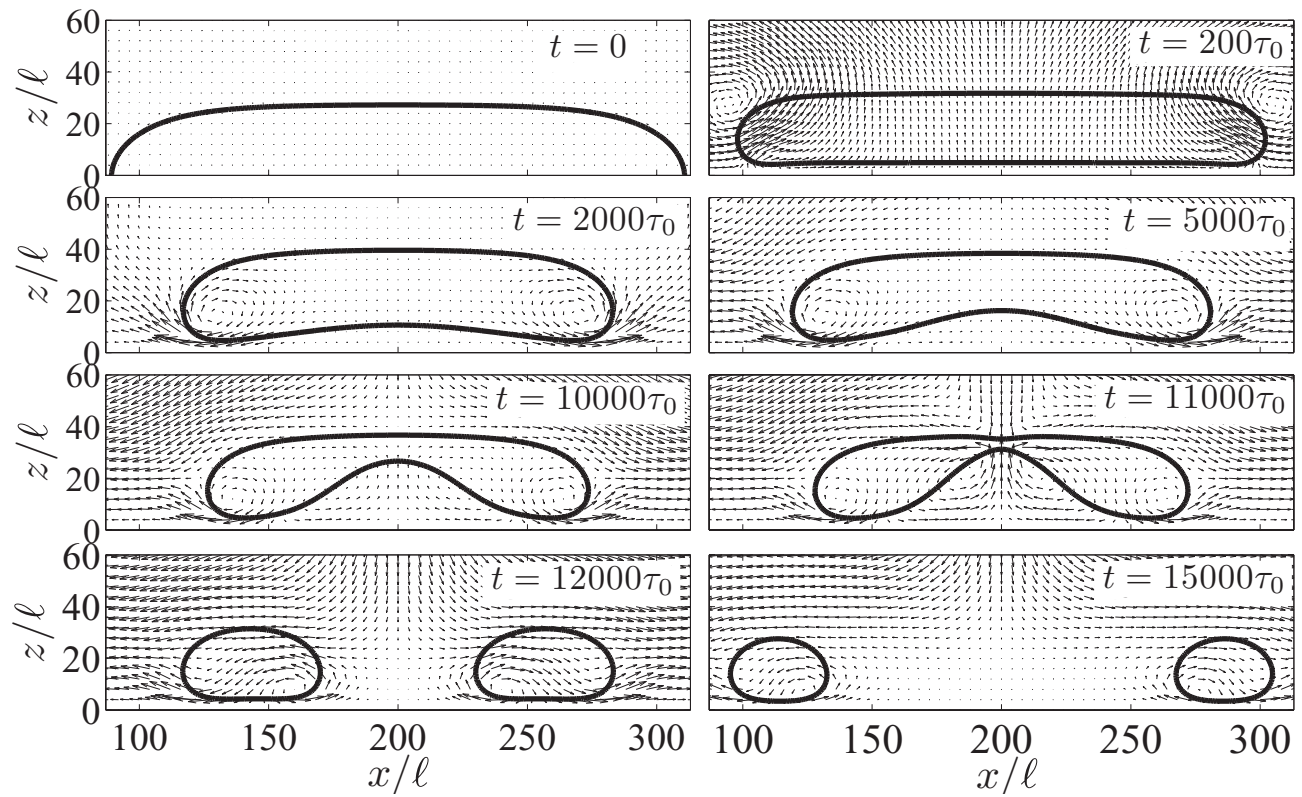

FIG. 2. Snapshots of droplet profiles (solid lines) and velocity fields (arrows) taken for a large evaporating puddle. The liquid-vapor interfaces are represented by the level curves of $n=\left(n_{l}+n_{v}\right) / 2$. A puddle of $r_{\text {max }}$ larger than the maximum size $R_{\max } \approx 4 R_{c}$ is unstable and breaks up into two smaller droplets. Here the dimensionless gravitational acceleration is $\mathcal{G}=1.0 \times 10^{-4}$, the capillary length is $R_{c} \approx 16 \ell$, the maximum size is $R_{\max } \approx 64 \ell$, and the system measures $L_{x}=400 \ell$ by $L_{z}=200 \ell$.

the geometry of the vapor layer $[5,15,16,20]$. Finally, we demonstrate the take-off of small circular droplets [15].

\section{A. Multiple length scales}

Consider a Leidenfrost droplet in two dimensions with mass $M$ (per length in the third direction). A vapor layer is stabilized between the droplet and the solid surface [5,15,16,20]. Assume that the droplet is large or heavy enough to stay close to the solid surface. Let $l$ be the horizontal extent of the vapor layer and $h$ be its thickness. The droplet is at mechanical equilibrium when

$$
M g \sim l \delta p,
$$

i.e., when its weight is balanced by the viscous pressure $\delta p$ of the vapor flow under the droplet. Although this pressure can be numerically obtained by solving the model in Sec. II A, it is worthwhile to present a scaling analysis for the vapor layer and the droplet above it. For this purpose, a few reasonable assumptions are made as follows [8,20].

(i) When $l \gg h$, the lubrication approximation is valid for describing the vapor flow between the droplet and the solid.

(ii) The vapor flow is incompressible, with

$$
u / l \sim w / h,
$$

where $u$ and $w$ are the characteristic horizontal velocity and the characteristic vertical velocity, respectively.

(iii) The flow in the thin vapor layer is a Poiseuille flow, driven by a horizontal pressure gradient of the order of magnitude of $\delta p / l$. This gives the momentum equation in the form of

$$
\delta p / l \sim \eta_{v} u / h^{2},
$$

where $\eta_{v}$ is the shear viscosity of the vapor. (iv) The temperature of the droplet is spatially uniform and fixed at the coexistence temperature $T_{\mathrm{cx}}$, and heat is transferred to the droplet only by conduction through the vapor layer $[5,8$, $15,20]$. From the Stefan condition for the energy conservation associated with the evaporation at the bottom surface of the droplet, the heat flux across the vapor layer is directly related to the evaporation rate:

$$
\lambda_{v} \delta T / h \sim \rho_{v} w L_{q},
$$

where $\lambda_{v}$ is the heat conductivity of the vapor, $\delta T$ is the temperature difference between the droplet and the solid surface, $\rho_{v}$ is the mass density of the vapor, and $L_{q}$ is the latent heat of evaporation per unit mass.

Using the relations (16) to (18), we find

$$
\delta p \sim \frac{\eta_{v} \lambda_{v} \delta T}{\rho_{v} L_{q}} \frac{l^{2}}{h^{4}}
$$

for the order of magnitude of the vapor pressure. Combining Eqs. (15) and (19), we obtain

$$
l^{3} / h^{4} \sim M / \rho_{l} R_{l}^{3},
$$

in which $\rho_{l}$ is the mass density of the liquid and $R_{l}$ is a characteristic length scale defined by

$$
R_{l} \equiv\left(\frac{\eta_{v} \lambda_{v} \delta T}{\rho_{v} \rho_{l} g L_{q}}\right)^{1 / 3} .
$$

The significance of this length will be discussed later.

Three different regimes can be identified according to the shape of the droplet $[5,15,19,20]$.

(i) Puddles [see Fig. 1(a) for a schematic illustration]: A Leidenfrost droplet, whose size is of the order of magnitude of $R_{c}$, takes the shape of a (disk- or pancakelike) puddle flattened by gravity. In this regime, the thickness $d$ of the puddle 
remains nearly constant. Determined by a balance between the interfacial free energy $(2 \gamma$ per unit area of the top and bottom surfaces of the droplet) and the gravitational potential energy ( $\rho_{l} g d^{2} / 2$ per unit area), the thickness of the puddle is given by $d=2 R_{c}$ [5]. Therefore, the mass $M$ of the puddle is of the order of magnitude of $\rho_{l} l d \sim \rho_{l} l R_{c}$ and the horizontal extent of the vapor layer $l$ is of the order of magnitude of the puddle radius $R$. It follows from Eq. (20) that

$$
h \sim\left(R_{l}^{3} / R_{c}\right)^{1 / 4} R^{1 / 2}
$$

which means that as evaporation proceeds, the droplet is shrinking (with the radius $R$ getting smaller) and sinking (with the vapor layer getting thinner). Note that Eq. (22) is also valid for three-dimensional puddles [5].

(ii) Quasicircular droplets [see Fig. 1(b) for a schematic illustration]: In this regime, the size of a droplet is measured by its radius $R$. The mass $M$ of the droplet is of the order of magnitude of $\rho_{l} R^{2}$ and the horizontal extent of the vapor layer $l$ is of the order of magnitude of $(R h)^{1 / 2}[15,20]$. It follows from Eq. (20) that

$$
h \sim R_{l}^{6 / 5} R^{-1 / 5}
$$

which means that as evaporation proceeds, the droplet is shrinking and rising (with the vapor layer getting thicker). Furthermore, for a droplet to remain quasicircular, the viscous pressure in the vapor layer should be comparable to the Laplace pressure in the droplet, i.e., $\delta p \sim \gamma / R$. This occurs when the droplet radius is of the order of magnitude of $R_{i}$, a characteristic length scale defined by

$$
R_{i} \equiv\left(R_{c}^{10} R_{l}^{3}\right)^{1 / 13}
$$

which is derived from $\delta p \sim \gamma / R_{i}$ by combining Eqs. (14), (19), (21), (23), and $l \sim(R h)^{1 / 2} \quad[15,20]$. Note that for three-dimensional droplets, Eq. (23) becomes $h \sim R_{l}^{3 / 2} R^{-1 / 2}$ and Eq. (24) becomes $R_{i} \equiv\left(R_{c}^{4} R_{l}^{3}\right)^{1 / 7}[15]$.

(iii) Circular droplets [see Fig. 1(c) for a schematic illustration]: For a droplet to remain circular, the viscous pressure in the vapor layer must be negligible compared to the Laplace pressure in the droplet, i.e., $\delta p \ll \gamma / R$ or, equivalently, $R \ll R_{i}$. In this regime, the scaling relation (23) derived for the quasicircular droplets above is still applicable.

It is interesting to note that the dependence of the vapor layer thickness $h$ on the droplet size $R$ changes qualitatively from Eq. (22) for puddles to Eq. (23) for quasicircular and circular droplets. That is, starting from a large puddle, (at least) two distinct stages can be identified in the course of evaporation, with a crossover occurring for $R \sim R_{i}$. A large evaporating puddle would shrink and sink [with a decreasing $h$ according to Eq. (22)] while a small evaporating droplet would shrink and rise [with an increasing $h$ according to Eq. (23)].

As emphasized in the beginning, the lubrication approximation is valid when $l \gg h$. It follows from Eq. (20) that this approximation breaks down when the droplet radius becomes comparable to the characteristic length scale $R_{l}$ defined by Eq. (21), with $l \sim h \sim R_{l}$ and $M \sim \rho_{l} R_{l}^{2}$. Physically, as the droplet radius $R$ becomes much smaller than $R_{l}$ due to continuous evaporation, the droplet takes off from the hot surface [15]. In other words, a Leidenfrost droplet of radius $R$ much larger than $R_{l}$ remains close to the solid surface, and, hence, the lubrication approximation is valid. Recently, it has been shown that the velocity and temperature fields in the vapor layer can be well described by the lubrication approximation for all cases with $R \gg R_{l}$ [20].

In summary, the above analysis shows that the characteristics of a Leidenfrost droplet are governed by its size in comparison with the characteristic length scales $R_{c}, R_{i}$, and $R_{l}$. They show up as the droplet size decreases in the course of evaporation. Some of the above scaling laws have been found to agree with the experimental data semiquantitatively [5,15,16,20]. In Ref. [5], the behavior described by Eq. (22) was observed for the vapor layer under puddles. In Ref. [15], the take-off of Leidenfrost droplets smaller than $R_{l}$ was demonstrated, and the behavior described by Eq. (23) was observed for the vapor layer under small droplets. In Ref. [16], Leidenfrost water drops were observed to show continuous shape change from a puddle to a quasisphere and to a sphere. Note that the capillary length $R_{c}=\left(\gamma / \rho_{l} g\right)^{1 / 2}$ defined by Eq. (14) is typically much larger than $R_{l}$ defined by Eq. (21). Therefore, we have $R_{i}=R_{c}^{10 / 13} R_{l}^{3 / 13} \gg R_{l}$, a condition realized in experiments [15]. In the present work, we use $\mathcal{R}=0.06, T_{\mathrm{cx}}=0.875 T_{c}, T_{w}=0.975 T_{c}$, and $\delta T \equiv$ $T_{w}-T_{\mathrm{cx}}$, with the estimations for the three length scales given by $R_{c} \approx 0.14 \ell / \mathcal{G}^{1 / 2}$ [in Eq. (14)], $R_{i} \approx 0.15 \ell / \mathcal{G}^{6 / 13}$, and $R_{l} \approx 0.19 \ell / \mathcal{G}^{1 / 3}$.

Finally, we would like to point out that in the previous theoretical scaling analyses [5,15,16,20], the above three length scales $R_{c}, R_{i}$, and $R_{l}$ are assumed to be well separated (with $R_{c} \gg R_{i} \gg R_{l}$ ) and the asymptotic behaviors are clearly manifested. However, for a simulation of the evaporation process starting from a large puddle, we can not afford to have a large separation of these length scales due to the limited computational capacity. In fact, we have to make use of the artificially large gravitational accelerations such that these length scales are no longer separated by orders of magnitude and, hence, simulations across these scales become affordable. Table I lists the values of these length scales for different dimensionless gravitational accelerations $\mathcal{G}$ used in Secs. III B and III C. For the purpose of reference, we also give the values of these length scales for water and nitrogen on the surface of the Earth in the appendix.

\section{B. Evaporation from puddles to circular droplets}

Here we present and discuss the numerical results obtained for evaporating Leidenfrost droplets that are large enough to remain close to the solid surface. We focus on the time evolution of the droplet shape and the geometry of the vapor layer in the course of evaporation $[5,15,16,20]$. To demonstrate the continuous shape change from a puddle to a circular droplet, we use artificially large gravitational accelerations such that $R_{c}$ and $R_{i}$ are not separated by order(s) of magnitude. This, of course, prevents us from displaying the various power laws [e.g., Eqs. (22) and (23)] obtained in the scaling analyses.

It has been experimentally observed that if the droplet is sufficiently large, then a vapor bubble (or even several bubbles for a very large puddle) rises at the center of the droplet and bursts when reaching the upper interface [5]. This arises from the Rayleigh-Taylor instability of the lower liquid-vapor interface. In our two-dimensional simulations shown in Fig. 2, 


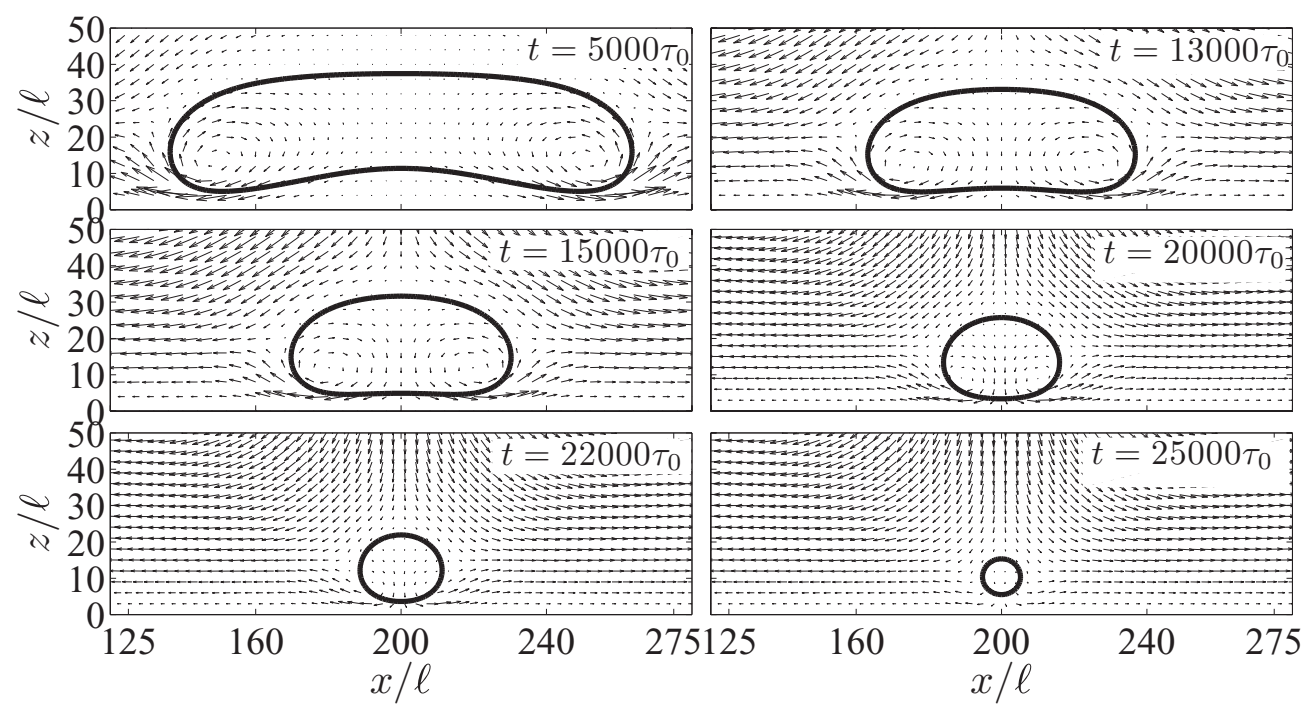

FIG. 3. Snapshots of droplet profiles (solid lines) and velocity fields (arrows) taken for an evaporating droplet that changes from a puddle to a circle. The liquid-vapor interfaces are represented by the level curves of $n=\left(n_{l}+n_{v}\right) / 2$. Here the dimensionless gravitational acceleration is $\mathcal{G}=1.0 \times 10^{-4}$, the capillary length is $R_{c} \approx 16 \ell$, and the other two characteristic lengths are $R_{i} \approx 11 \ell$ and $R_{l} \approx 4 \ell<R_{\min }=5 \ell$. The system measures $L_{x}=400 \ell$ by $L_{z}=200 \ell$. Note that the vapor flows above the droplet are caused by the Rayleigh-Bénard convection with the Rayleigh number $\mathrm{Ra} \sim 1000$.

it is also observed that if the maximum radius $r_{\max }$ of a puddle is larger than $4.5 R_{c}$, then the vapor pocket beneath the droplet rises continuously to the top of the droplet and bursts. Consequently, the droplet breaks up into two smaller droplets, which quickly run away from each other due to the fast transformation of the interfacial energy of the mother droplet before the breakup into the kinetic energy of the daughter droplets after the breakup.

Regardless of the initial size of the droplet, the formation of the vapor layer between the droplet and the solid is very fast (from $t=0$ to $t=200 \tau_{0}$ in Fig. 2). This may be attributed to the fact that in the present mean-field approach, the vapor layer is formed via the instability of the superheated liquid near the solid surface. That is, the vapor layer is formed via spinodal decomposition rather than discrete bubble nucleation events, which are thermodynamic fluctuations excluded from our model.

Below we present the numerical results for evaporation processes that start from large puddles (with $r_{\max }$ being a bit smaller than $R_{\max }$ ). The main findings may be summarized as follows.

(i) As evaporation proceeds, the droplet shape changes continuously from puddles to quasicircular droplets and to circular droplets (see Fig. 3). Figure 4 shows that the droplet shape is controlled by its instantaneous size in comparison with the two characteristic length scales $R_{c}$ and $R_{i}$. A droplet of radius $r_{\max }$ larger than $R_{c}$ (from $t=0$ to $t \approx 20000 \tau_{0}$ ) takes the shape of puddles flattened by gravity. In particular, the puddle thickness $d$ remains nearly constant $\left(\approx 2 R_{c}\right)$ from $t=5000 \tau_{0}$ to $t=15000 \tau_{0}$, as seen in Fig. 3 and Fig. 4. The latter shows the time dependence of $r_{\min }=d / 2$. As the decreasing radius $r_{\max }$ approaches $R_{i}$, the droplet shape becomes quasicircular and then circular. This is displayed by the three droplet radii $r_{\min }, r_{\max }$, and $r_{\text {eff }}$ that merge when they become small enough [see Fig. 4(a)]. Here $r_{\text {eff }}$ is defined by $\pi r_{\text {eff }}^{2}=$ the instantaneous droplet area. These results are consistent with the above scaling analysis. The nearly constant puddle thickness $d \approx 2 R_{c}$ seen in our simulations agrees with the experimental observation [5].

(ii) Figure 3 shows that vortices are generated within the droplet in the course of evaporation. The generation of vortices in the Leidenfrost droplets is anticipated because vortices are also observed in evaporating droplets in direct contact with solid surfaces, through simulations [28,33] and experiments $[11,55]$. It is interesting to note that Fig. 3 shows large vapor flows above the droplet. They are induced by the RayleighBénard convection in the dense vapor phase (sandwiched between the hot bottom substrate with $T_{w}=0.975 T_{c}$ at $z=0$ and the cold top substrate with $T_{w}=0.875 T_{c}$ at $z=L_{z}=$

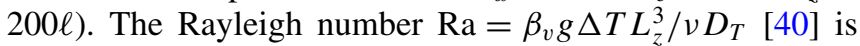
found to be of the order of magnitude of 1000 , where $\beta_{v} \equiv-1 /\left[\rho(\partial T / \partial \rho)_{p}\right]_{\rho=\rho_{v}, p=p_{\mathrm{cx}}}$ is the thermal-expansion coefficient of the vapor and $\Delta T=0.1 T_{c}$ is the temperature difference between the two substrates. (In our simulations, $\mathcal{R} \equiv v^{2} m / \varepsilon \ell^{2}=0.06, \mathcal{G} \equiv m g \ell / \varepsilon=10^{-4}, \operatorname{Pr} \equiv v / D_{T} \sim 1$, $\beta_{v} T_{c} \sim 1$ at the coexistence pressure $p_{\mathrm{cx}} \approx 0.584 p_{c}$ ).

(iii) The dynamic van der Waals theory describes the one-component fluids in which the liquid and vapor are pure, consisting of only one molecular component. As a consequence, the temperature is nearly a constant along the liquid-vapor interface [21,22]. This has been observed in our simulations. For example, in Fig. 3 with the hot bottom substrate at $0.975 T_{c}$ and the cold top substrate at $0.875 T_{c}$, the temperature at the liquid-vapor interface is found to be everywhere close to $0.92 T_{c}$. Furthermore, the liquid droplet enclosed by this interface shows a very small temperature variation as well. As to the temperature distribution in the vapor layer, our numerical simulations show that the temperature decreases monotonically from the heated bottom substrate to the liquid-vapor interface. In fact, this distribution can be well 

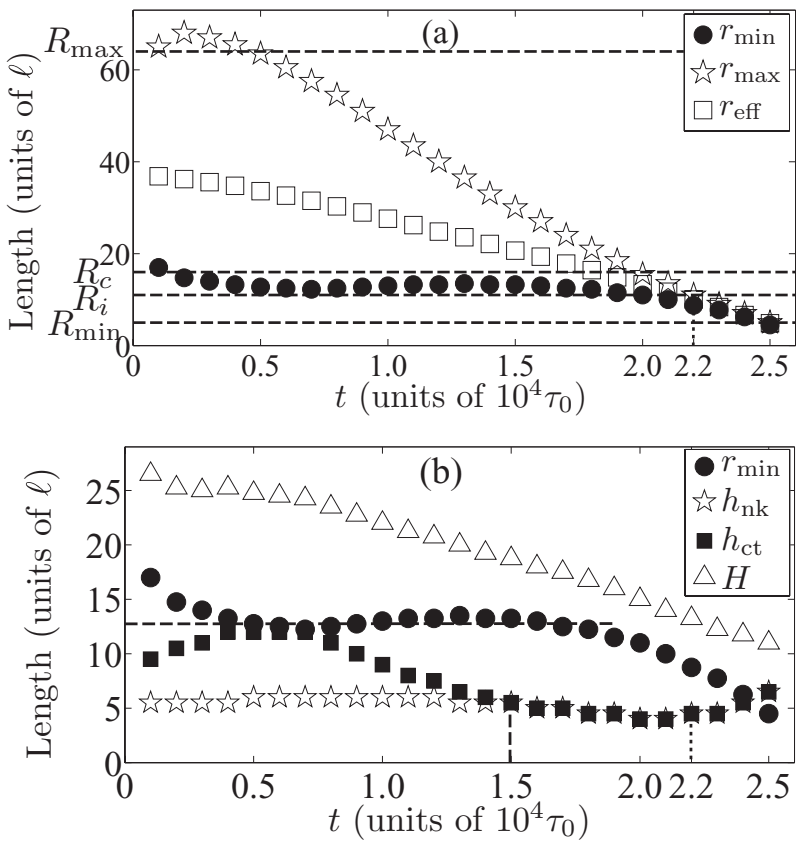

FIG. 4. Temporal evolution of the droplet in Fig. 3. Here various lengths associated with the droplet (see Fig. 1) are plotted as functions of time $t$ (with $r_{\text {eff }}$ defined by $\pi r_{\text {eff }}^{2}=$ the instantaneous droplet area). (a) The four horizontal dashed lines mark the four characteristic length scales $R_{\max }, R_{c}, R_{i}$, and $R_{\min }$. The vertical dotted line marks the time $\left(t=22000 \tau_{0}\right)$ at which the three radii $r_{\min }, r_{\max }$, and $r_{\text {eff }}$ merge, i.e., the droplet becomes circular as seen in Fig. 3. (b) The horizontal dashed line is used to indicate that the radius $r_{\text {min }}$ (or the thickness $d=2 r_{\min }$, see Fig. 1) almost remains constant from $t=5000 \tau_{0}$ to $15000 \tau_{0}$ in the stage of an evaporating puddle. The vertical dashed line marks the time $\left(t=15000 \tau_{0}\right)$ at which the bottom surface of the droplet becomes flat with $h_{\mathrm{ct}}=h_{\mathrm{nk}}$. The vertical dotted line still marks the time at which the droplet becomes circular. After that, the vapor layer gets thicker as evaporation proceeds.

approximated by $\nabla \cdot(\lambda \nabla T)=0$ because the Péclet number is small $(\sim 0.1)$ and the vapor flow is nearly orthogonal to the temperature gradient.

As explained before, artificially large gravitational accelerations have to be used to make the simulations computationally affordable. In order to make our results consistent and convincing, we have carried out simulations using several different gravitational accelerations. Figure 5 shows the time dependence of the vapor layer thickness $h_{\mathrm{ct}}$ (defined in Fig. 1) for four gravitational accelerations. The quantitatively similar characteristics are as follows:

(i) A puddle of radius larger than $R_{i}$ evaporates with the vapor layer getting thinner, as displayed by the decreasing $h_{\mathrm{ct}}$ with decreasing radius $r_{\max }$. This is in qualitative agreement with the scaling relation (22).

(ii) A quasicircular or circular droplet, which is of radius equal to or smaller than $R_{i}$, evaporates with the vapor layer getting thicker, as displayed by the increasing $h_{\mathrm{ct}}$ with decreasing radius $r_{\max }$. This is in qualitative agreement with the scaling relation (23).

It is noted that, in all four cases, the crossover between the two qualitatively different evaporation stages occurs when the

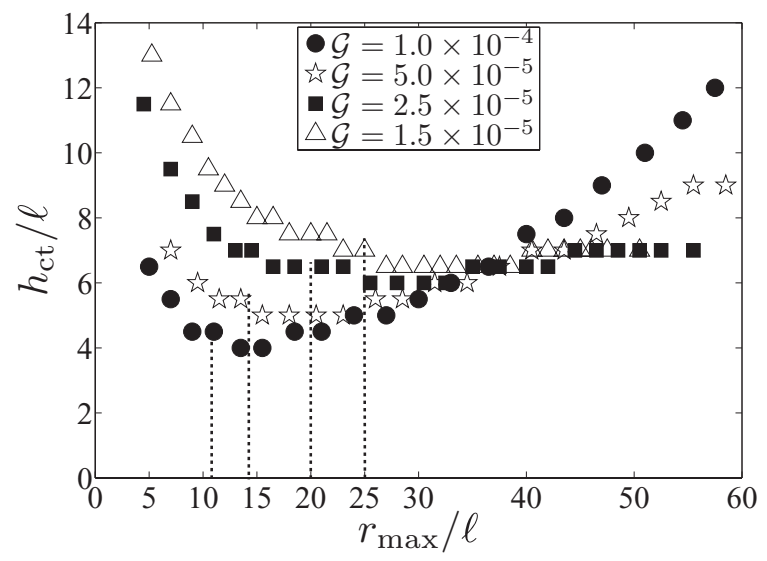

FIG. 5. The central thickness of the vapor layer $h_{\mathrm{ct}}$ (defined in Fig. 1) is plotted as a function of the droplet radius $r_{\max }$ for an evaporating droplet that changes from a puddle to a circle. Four different dimensionless gravitational accelerations $\mathcal{G}$ have been used. Each vertical dotted line marks the value of $R_{i}$ corresponding to a particular value of $\mathcal{G}$ (see Table I). All the four sets of data show that $h_{\mathrm{ct}}$ starts to increase with the decreasing $r_{\max }$ as soon as the droplet radius becomes smaller than $R_{i}$.

droplet radius approaches $R_{i}$. This observation agrees with the predication of the scaling analysis in Sec. III A.

We have also studied the effects of substrate temperature on the droplet shape and the geometry of the vapor layer. Figures 6(a)-6(c) show the three radii $r_{\mathrm{eff}}, r_{\mathrm{nk}}$, and $r_{\text {min }}$ (defined in Fig. 1), each plotted as a function of the radius $r_{\max }$ for five different bottom substrate temperatures $T_{w}$ (and, thus, different evaporative fluxes) in the course of evaporation. It is readily seen that these three radii, by which the droplet shape is characterized, mainly depend on $r_{\max }$ and are nearly independent of $T_{w}$ [16]. Figures 6(d) and 6(e) show the two vapor layer thicknesses $h_{\mathrm{ct}}$ and $h_{\mathrm{nk}}$ (defined in Fig. 1), each plotted as a function of the radius $r_{\max }$ for five different bottom substrate temperatures $T_{w}$ in the course of evaporation. These two thicknesses, by which the geometry of the vapor layer is characterized, mostly depend on $r_{\max }$ and are nearly independent of $T_{w}$ in the range from $0.975 T_{c}$ to $1.0 T_{c}$. These numerical results agree with the recent experimental observations that the geometry of the vapor pocket depends primarily on the drop size and not on the substrate temperature [16]. At a very high substrate temperature $T_{w}=1.1 T_{c}$, however, $h_{\mathrm{ct}}$ and $h_{\mathrm{nk}}$ show an appreciable increase in magnitude. Physically, a higher substrate temperature leads to stronger evaporation at the bottom surface of the droplet, and, consequently, the droplet is pushed higher and the vapor layer gets thicker.

It is interesting to note that in the initial stage of the evaporation, the neck radius of the puddle is found to be $r_{\mathrm{nk}} \approx$ $r_{\max }-0.6 R_{c}$ [as represented by the solid line in Fig. 6(b)], in reasonable agreement with the previous theoretical results [19] and the experimental observations that the maximum radius and the neck radius are related by $r_{\mathrm{nk}} \approx r_{\max }-0.5 R_{c}$ [16].

We would like to point out that, to produce the above results, artificially large gravitational accelerations have been used. In particular, they are chosen to be so large that $R_{l}$ becomes close to $R_{\min }$, as shown in Table I. As a result, the take-off of small circular droplets, which is predicted to occur when the 


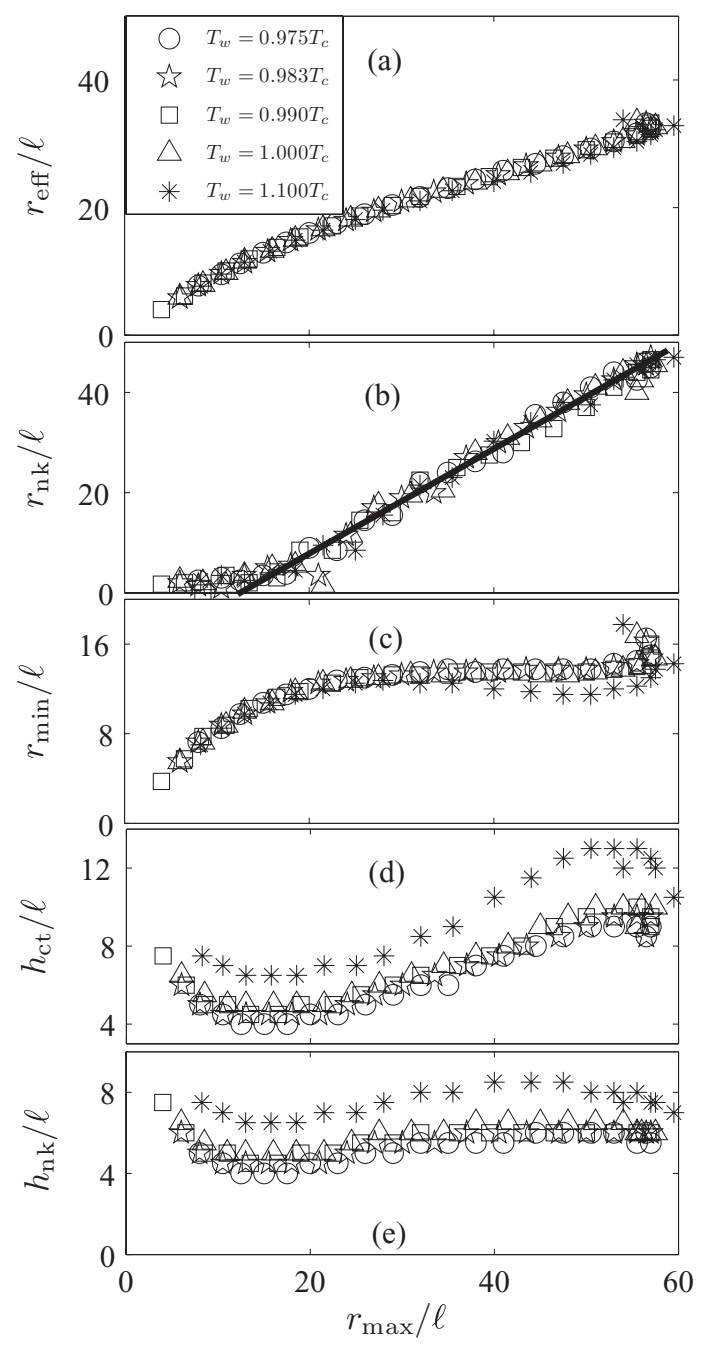

FIG. 6. Various lengths associated with the droplet (see Fig. 1) are plotted as functions of the instantaneous radius $r_{\max }$ for an evaporating droplet that changes from a puddle to a circle. Five different substrate temperatures $T_{w}$ have been used. Here $r_{\text {eff }}$ is defined by $\pi r_{\text {eff }}^{2}=$ the instantaneous droplet area, and the dimensionless gravitational acceleration is $\mathcal{G}=1.0 \times 10^{-4}$. The solid line in (b) is a linear least-squares fitting of $r_{\mathrm{nk}}$, for $r_{\max }$ from $20 \ell$ to $60 \ell$. This gives $r_{\mathrm{nk}} \approx r_{\max }-0.6 R_{c}$ with $R_{c}=16 \ell$.

droplet radius becomes much smaller than $R_{l}$, is beyond the access of numerical simulations. This is actually indicated by the monotonic decrease of the droplet height $H \equiv h_{\mathrm{ct}}+r_{\min }$ in the course of evaporation [see Fig. 4(b)]. Therefore, in order to observe the take-off of small circular droplets, we have to use smaller gravitational accelerations that make $R_{l}$ much larger than $R_{\min }$.

\section{Take-off of small circular droplets}

Celestini et al. [15] predicted and observed that, below the critical size $R_{l}$, a small spherical Leidenfrost droplet would take off from the hot solid surface. In this regime, the levitating force generated by the vapor pressure can no longer be balanced by the diminished weight of the droplet. To numerically investigate the transient dynamics of the take-off, we have performed simulations by using artificially large gravitational accelerations $(\mathcal{G})$ which make $R_{l} \sim 20 \ell$ (as shown in Table I). The take-off, predicted for droplets smaller than $R_{l}$ but larger than $R_{\text {min }}=5 \ell$, therefore becomes numerically accessible. The main findings may be summarized as follows.

(i) A droplet of radius smaller than $R_{l}\left(\ll R_{i}\right)$ takes the circular shape as displayed in the upper panel of Fig. 7 and also indicated by the merging of three droplet radii $r_{\min }, r_{\max }$, and $r_{\text {eff }}$ in the lower panel of Fig. 7. As evaporation proceeds, the vapor layer under the droplet becomes thicker, as shown by $h_{\mathrm{ct}}$ and $h_{\mathrm{nk}}$ in the lower panel of Fig. 7, in consistency with the scaling relation (23) for droplets smaller than $R_{i}$.

(ii) The droplet radius is a linear decreasing function of time, as shown by $r_{\min }=r_{\max }=r_{\mathrm{eff}}$ in the lower panel of Fig. 7. This means that, in our two dimensions, the rate of evaporation is almost a constant independent of the droplet size. This is consistent with the fact that in onecomponent fluids the droplet surface is almost isothermal at the coexistence temperature [22,33].

(iii) For easy recognition, the beginning of the droplet takeoff is marked by the start of the increase of the droplet height $H \equiv h_{\mathrm{ct}}+r_{\text {min }}$ (defined in Fig. 1). Figure 7 shows that the droplet starts to take off at $t=90000 \tau_{0}$ with a radius smaller than $R_{l}$.

(iv) The upper panel of Fig. 7 shows the velocity fields before and after the take-off. The velocity magnitude in the droplet is smaller than that in the surrounding vapor due to the viscosity contrast. In particular, above the droplet there is a circulating flow and below there is a jet flow. Together, they result in an upward force on the droplet.

(v) As explained before, artificially large gravitational accelerations have to be used to make the simulations affordable. In order to make our results consistent and convincing, we have carried out simulations using four different gravitational accelerations. Figure 8 shows that for each gravitational acceleration, the droplet starts to take off (with $H$ increasing with the decreasing $r_{\max }$ ) when its radius falls below $R_{l}$. In particular, the smaller the gravitational acceleration is (i.e., the closer to the real one on the surface of the Earth), the more slowly the droplet height $H$ decreases before the take-off and the more rapidly $H$ increases after the take-off. It is, therefore, reasonable to believe that in the real situation on the surface of the Earth, the droplet height would remain almost constant before the take-off and increase very rapidly after the take off. This agrees with the experimental observation of the take-off of water droplets on a silicon wafer (see the lower panel of Fig. 2 in Ref. [15]).

(vi) It is hereby noted that, for $\mathcal{G}=2.5 \times 10^{-7}$ (the smallest in Fig. 8), the decrease of $H$ with the decreasing $r_{\max }$ before the take-off is not shown. This is because the initial size of the droplet is not large enough, a limitation of our computational capability. As a result, from the very beginning of the simulation, the droplet weight is already too small compared to the levitating force generated by the vapor pressure. Consequently, the droplet takes off immediately.

\section{CONCLUDING REMARKS}

We have investigated the hydrodynamics of Leidenfrost droplets in two-dimensional space by employing the dynamic 

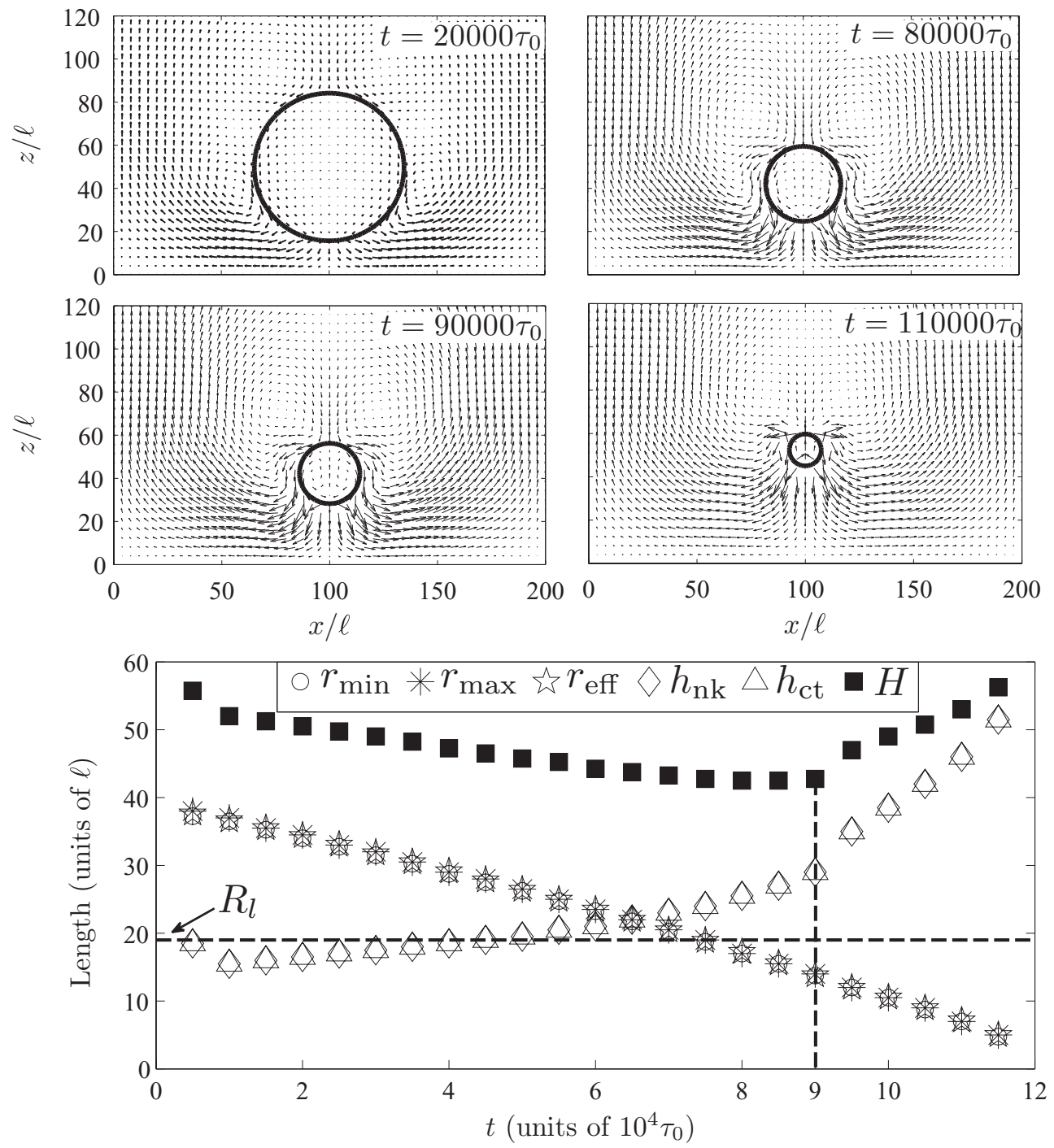

FIG. 7. Upper panel: Snapshots of droplet profiles (solid lines) and velocity fields (arrows) taken for a small evaporating droplet. The liquid-vapor interfaces are represented by the level curves of $n=\left(n_{l}+n_{v}\right) / 2$. Lower panel: To show the temporal evolution of the droplet in the upper panel, various lengths (see Fig. 1) are plotted as functions of time $t$. The horizontal dashed line marks the characteristic length scale $R_{l} \approx 19 \ell\left(\gg R_{\min }=5 \ell\right)$. Here the dimensionless gravitational acceleration is $\mathcal{G}=1.0 \times 10^{-6}$, and the system measures $L_{x}=200 \ell$ by $L_{z}=300 \ell$. The vertical dashed line marks the time after which the droplet height $H$ (defined in Fig. 1) starts to increase. This signals the take-off of the droplet at $t=90000 \tau_{0}$. Note that the three radii $r_{\min }, r_{\max }$, and $r_{\mathrm{eff}}$ merge, and the droplet is circular. The thicknesses $h_{\mathrm{ct}}$ and $h_{\mathrm{nk}}$ are equal and increase with time, in agreement with Eq. (23) for droplets smaller than $R_{i}=88 \ell$.

van der Waals theory [21,22], which is a phase-field model capable of describing the two-phase hydrodynamics involving the liquid-vapor transition in inhomogeneous temperature field. We have studied the evolution of the evaporating droplets which are puddles initially and become circles gradually.

Our results have shown that a Leidenfrost droplet of radius $r_{\max }$ larger than $R_{\max } \approx 4 R_{c}$ is unstable and breaks up into smaller droplets (see Fig. 2) due to the Rayleigh-Taylor instability of the bottom surface of the droplet $[5,19]$. Our results have demonstrated that in the evolution of a droplet from a puddle to a circle, two characteristic length scales are manifested: the capillary length $R_{c} \equiv\left(\gamma / \rho_{l} g\right)^{1 / 2}$ defined in Eq. (14) and $R_{i} \equiv\left(R_{c}^{10} R_{l}^{3}\right)^{1 / 13}$ defined in Eq. (24). A droplet of radius $r_{\max } \sim R_{c}$ takes the shape of a puddle as shown in Fig. 3. As the decreasing radius $r_{\max }$ approaches $R_{i}$, the droplet shape becomes more and more circular as shown in Fig. 3 and Fig. 4. As to the evolution of the geometry of the vapor layer under the droplet, it has been numerically observed that a droplet of radius larger than $R_{i}$ evaporates with the vapor layer getting thinner as shown in Fig. 5 while a quasicircular or circular droplet of radius equal to or smaller than $R_{i}$ evaporates with the vapor layer getting thicker as shown in Fig. 5. These observations are consistent with the predictions of scaling analyses $[15,16,20]$. In addition, our numerical results have shown agreement with the experimental observation [16] that the geometry of the vapor layer mostly depends on the droplet size and is almost independent of the substrate temperature $T_{w}$. Finally, our numerical results have displayed that, as theoretically predicted and experimentally observed [15], a circular droplet of radius smaller than the 


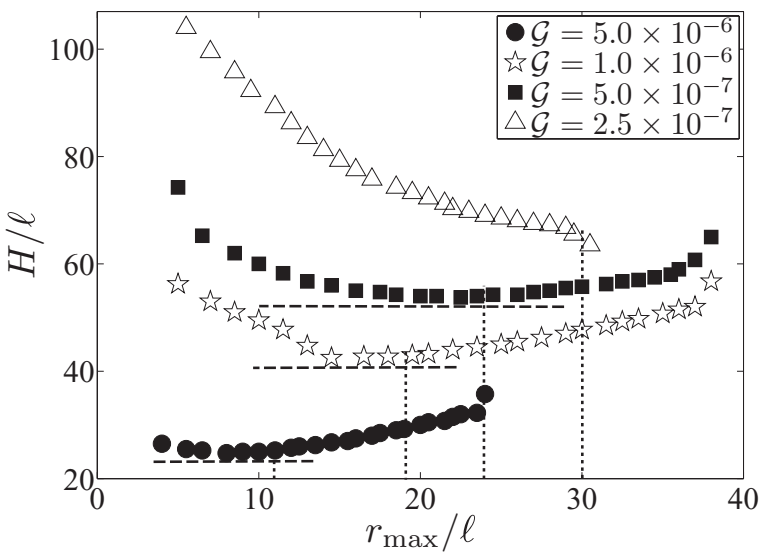

FIG. 8. The droplet height $H$ (defined in Fig. 1) is plotted as a function of the droplet radius $r_{\max }$ for an evaporating droplet. Four different dimensionless gravitational accelerations $\mathcal{G}$ have been used. Each horizontal dashed line indicates a minimum of $H$ that signals the beginning of a take-off. Each vertical dotted line marks the value of $R_{l}$ corresponding to a particular value of $\mathcal{G}$ (see Table I). The four sets of data show the trend that the smaller $\mathcal{G}$ is, the more slowly $H$ decreases before the take-off and the more rapidly $H$ increases after the take-off.

characteristic length $R_{l}$ [defined in Eq. (21)] takes off from the hot substrate, as shown in Fig. 7 and Fig. 8. (In our two-dimensional simulations, $R_{l}$ is much smaller than $R_{i}$, as noted in Table I.) The consistency of our simulation results with recent theoretical predictions and experimental observations shows the dynamic van der Waals theory to be an effective and efficient model for studying the hydrodynamics of Leidenfrost droplets and related systems.

Below we make a few concluding remarks.

(i) Because the mesh size $\Delta x=\Delta z=0.5 \ell$ is only a few angstroms [22,34], the simulated systems are very small (about a few hundreds of nanometers) and the droplets are as large as a few tens of nanometers. In these small systems, gravity plays no role. In order to induce appreciable gravitational effects, artificially large gravitational accelerations have been used. As a result, our simulations can only provide qualitative and semiquantitative understanding for the real dynamics of Leidenfrost droplets at millimeter scale under the gravity on the surface of the Earth. We would like to point out that more realistic simulations at larger length scales can be implemented with the help of the adaptive mesh method [49].

(ii) Possible effects of the wettability and the finite heat conductivity of the solid substrates have not been discussed. Moreover, boundary velocity slip, temperature slip (Kapitza resistance), and mechanical-thermal cross coupling at the fluid-solid interface $[41-44,46]$ have been completely excluded from our model. These simplifications are expected not to qualitatively affect the behaviors of the Leidenfrost droplets that are separated from the solid by the vapor $[3,20,47]$. Nevertheless, we would like to point out that a detailed modeling of the physical processes at the fluid-solid interface does become essential when we study the formation of the vapor layer $[1,18]$, the determination the Leidenfrost temperature $[1,18]$, and the self-propelled motion of the Leidenfrost droplets on ratchets [56]. (iii) All the fluctuation effects have been neglected. However, it has been experimentally observed for Leidenfrost droplets that strong fluctuations exist in the central vapor pocket and around the neck region [16]. These fluctuations are fast in both time and space and may have important effects on droplet propulsion and oscillation and the star drops observed at high substrate temperatures [12].

(iv) Heat transfer from the solid to the droplet is realized not only by conduction across the vapor layer but also by radiation from the hot surface [8]. This is particularly the case when the substrate temperature is further increased above the Leidenfrost temperature. Therefore, radiation should be taken into account by a more complete model.

(v) Leidenfrost hydrodynamics in binary fluid mixtures is an interesting topic to pursue $[18,22,57,58]$. In these systems, the Marangoni effects may decisively govern the droplet dynamics even at small solute concentrations [59].

\section{ACKNOWLEDGMENTS}

This publication is based on work partially supported by Award No. SA-C0040/UK-C0016, made by King Abdullah University of Science and Technology (KAUST), and Hong Kong RGC Grant No. 603510.

\section{APPENDIX: MATERIAL PARAMETERS}

In this appendix, we calculate and estimate various parameters used in the model from the available material parameters of water [60] and nitrogen $[1,61]$. We want to point out that the dynamic van der Waals theory is employed to model the Leidenfrost droplets with a small set of material parameters: the molecular mass $m$, the energy parameter $\varepsilon$, the volume parameter $v_{0}$, the coefficient $C$ for the gradient contribution to entropy density, and the kinematic viscosity $v$. All the other material parameters are expressed in terms of these five basic parameters. Below we show that the basic parameters can be estimated using the available material parameters (of water and nitrogen). This means that for water and nitrogen at $T_{\mathrm{cx}} \approx 0.9 T_{c}$, the various material properties (equilibrium and nonequilibrium) can be described by the dynamic van der Waals theory semiquantitatively. We therefore are encouraged to use this theory with the understanding that the material parameters used are close to their realistic values. On the other hand, the gravitational acceleration $g$ is not a material property. It measures the strength of the external force. Artificially large values have been used for $g$ to make the dimensionless length scales $R_{c} / \ell, R_{i} / \ell$, and $R_{l} / \ell$ small enough to meet our computational capability.

(i) The molecular volume $v_{0}=a_{\mathrm{vdw}}^{3}$ and the energy parameter $\varepsilon$ can be calculated from the critical properties. As described in Sec. II A, the van der Waals fluids are characterized by these two parameters. From the critical properties in Table II, we

TABLE II. Parameters for water [60] and nitrogen [61].

\begin{tabular}{lcccc}
\hline \hline Substance & $\mathrm{m}(\mathrm{g} / \mathrm{mol})$ & $T_{c}(\mathrm{~K})$ & $p_{c}(\mathrm{MPa})$ & $n_{c}\left(10^{28} / \mathrm{m}^{3}\right)$ \\
\hline Water & 18 & 647.1 & 322.0 & 1.1 \\
Nitrogen & 28 & 126.2 & 3.4 & 0.7 \\
\hline \hline
\end{tabular}


TABLE III. Properties of water [60] and nitrogen $[1,61]$ at a given temperature. The subscripts $l$ and $v$ are used to denote the liquid and vapor phases at coexistence.

\begin{tabular}{lcc}
\hline \hline Substance & Water & Nitrogen \\
\hline$T_{\mathrm{cx}}(\mathrm{K})$ & 603 & 115 \\
$p_{\mathrm{cx}}(\mathrm{MPa})$ & 12.9 & 1.94 \\
$n_{l}\left(10^{28} / \mathrm{m}^{3}\right)$ & 2.14 & 1.25 \\
$n_{v}\left(10^{28} / \mathrm{m}^{3}\right)$ & 0.26 & 0.19 \\
$\gamma(\mathrm{mN} / \mathrm{m})$ & 7.7 & 1.2 \\
$\eta_{l}(\mu \mathrm{Pa} \mathrm{s})$ & 74.6 & 59.9 \\
$\eta_{v}(\mu \mathrm{Pa} \mathrm{s})$ & 21.6 & 9.3 \\
$\lambda_{l}(\mathrm{~mW} / \mathrm{K} \mathrm{m})$ & 489.2 & 70.4 \\
$\lambda_{v}(\mathrm{~mW} / \mathrm{K} \mathrm{m})$ & 94.48 & 20.8 \\
$D_{T l}\left(10^{-6} \mathrm{~m}^{2} / \mathrm{s}\right)$ & 0.11 & 0.04 \\
$D_{T v}\left(10^{-6} \mathrm{~m}^{2} / \mathrm{s}\right)$ & 0.13 & 0.09 \\
$L_{q}(\mathrm{~kJ} / \mathrm{kg})$ & 1140.3 & 116.2 \\
$\beta_{l}\left(10^{-3} / \mathrm{K}\right)$ & 5.5 & 20.1 \\
$\beta_{v}\left(10^{-3} / \mathrm{K}\right)$ & 11.9 & 34.9 \\
\hline \hline
\end{tabular}

can calculate $\varepsilon=27 k_{B} T_{c} / 8$ and $v_{0}=1 / 3 n_{c}$ for the van der Waals fluids. For water, we have $\varepsilon \approx 3.0 \times 10^{-20} \mathrm{~J}$ and $v_{0} \approx$ $30 \times 10^{-30} \mathrm{~m}^{3}$, from which we obtain $a_{\mathrm{vdw}} \equiv v_{0}^{1 / 3} \approx 4 \AA$. For nitrogen, we have $\varepsilon \approx 1.0 \times 10^{-20} \mathrm{~J}$ and $v_{0} \approx 50 \times 10^{-30} \mathrm{~m}^{3}$, from which we obtain $a_{\mathrm{vdw}} \approx 4 \AA$.

(ii) The characteristic interfacial thickness $\ell$ can be calculated from the surface tension $\gamma$. As described in Sec. II A, the surface tension can be estimated by Eq. (7). Substituting the experimental value (in Table III) into this equation, we obtain $\ell \approx\left(\gamma v_{0} / k_{B} T\right)\left(1-T / T_{c}\right)^{-3 / 2}$. For water at the coexistence temperature $T_{\mathrm{cx}}=603 \mathrm{~K}$, we have $\ell \approx 16 \AA \approx 4 a_{\mathrm{vdw}}$. For nitrogen at $T_{\mathrm{cx}}=115 \mathrm{~K}$, we have $\ell \approx 3 \AA \approx a_{\mathrm{vdw}}$. It is seen that $\ell / a_{\mathrm{vdw}} \sim 1$ for both water and nitrogen. (iii) The product of the critical temperature $T_{c}$ and the thermal expansion coefficient $\beta$ is of order unity for either liquid or vapor (see Table III). For water at $T_{\mathrm{cx}}=603 \mathrm{~K}$, we have $\beta_{l} T_{c} \approx 3.6$ for the liquid and $\beta_{v} T_{c} \approx 7.7$ for the vapor. For nitrogen at $T_{\mathrm{cx}}=115 \mathrm{~K}$, we have $\beta_{l} T_{c} \approx 2.5$ and $\beta_{v} T_{c} \approx 4.4$. This fact is reflected in the calculations for the van der Waals fluids (see Sec. III B).

(iv) The liquid-to-vapor ratios for the density, viscosity, and heat conductivity are approximately 5 at the coexistence temperature $T_{\mathrm{cx}} \approx 0.9 T_{c}$ (see Table III). For water at $T_{\mathrm{cx}}=$ $603 \mathrm{~K}$, the density ratio is $n_{l} / n_{v} \approx 8.3$, the viscosity ratio is $\eta_{l} / \eta_{v} \approx 3.5$, and the heat conductivity ratio is $\lambda_{l} / \lambda_{v} \approx$ 5.2. For nitrogen at $T_{\mathrm{cx}}=115 \mathrm{~K}$, we have $n_{l} / n_{v} \approx 6.7$, $\eta_{l} / \eta_{v} \approx 6.4$, and $\lambda_{l} / \lambda_{v} \approx 3.4$. These values are all around 5 . This fact is reflected in our assumptions $\eta=v m n$ and $\lambda=$ $v k_{B} n$ with the density ratio $n_{l} / n_{v} \approx 5$ at $T=0.875 T_{c}$ (see Sec. II B).

(v) For either water or nitrogen, the Prandtl number $\mathrm{Pr}=$ $v / D_{T}$ is of order unity in both liquid and vapor (see Table III). For water, we have $\operatorname{Pr}_{l}=1.1$ in the liquid and $\operatorname{Pr}_{v}=2.2$ in the vapor. For nitrogen, we have $\operatorname{Pr}_{l}=2.7$ and $\operatorname{Pr}_{v}=1.2$. This fact is reflected in our simulations through the use of $\eta=v m n$ and $\lambda=v k_{B} n$ (see Sec. II B).

(vi) The dimensionless parameters $\mathcal{R} \equiv v^{2} m / \varepsilon \ell^{2}$ and $\mathcal{G} \equiv$ $m g \ell / \varepsilon$ can be computed from the above parameter values. For water, we have $\mathcal{R}_{l} \approx 0.01$ in the liquid, $\mathcal{R}_{v} \approx 0.03$ in the vapor, and $\mathcal{G} \approx 1.6 \times 10^{-14}$. For nitrogen, we have $\mathcal{R}_{l} \approx$ $\mathcal{R}_{v} \approx 0.5$ and $\mathcal{G} \approx 2.1 \times 10^{-14}$.

(vii) The characteristic length scales $R_{c}, R_{i}$, and $R_{l}$ can be computed from the above parameters as well. For water, we have $R_{c} \equiv\left(\gamma / \rho_{l} g\right)^{1 / 2} \approx 3.9 \mathrm{~mm}, R_{i} \equiv\left(R_{c}^{10} R_{l}^{3}\right)^{1 / 13} \approx$ $0.9 \mathrm{~mm}$, and $R_{l} \equiv\left(\eta_{v} \lambda_{v} \delta T / \rho_{v} \rho_{l} g L_{q}\right)^{1 / 3} \approx 6.2 \mu \mathrm{m}$. For nitrogen, we have $R_{c} \approx 0.5 \mathrm{~mm}, R_{i} \approx 0.2 \mathrm{~mm}$, and $R_{l} \approx 3.5 \mu \mathrm{m}$.
[1] V. P. Carey, Liquid-Vapor Phase-Change Phenomena (Taylor \& Francis, New York, 2008).

[2] V. K. Dhir, Annu. Rev. Fluid Mech. 30, 365 (1998).

[3] D. Quéré, Annu. Rev. Fluid Mech. 45, 197 (2012).

[4] J. G. Leidenfrost, De Aquae Communis Nonnullis Qualitatibus Tractatus (Impensis Hermanni Ovenni, Duisburg, 1756).

[5] A.-L. Biance, C. Clanet, and D. Quéré, Phys. Fluids 15, 1632 (2003).

[6] I. U. Vakarelski, J. O. Marston, D. Y. C. Chan, and S. T. Thoroddsen, Phys. Rev. Lett. 106, 214501 (2011).

[7] T. Tran, H. J. J. Staat, A. Prosperetti, C. Sun, and D. Lohse, Phys. Rev. Lett. 108, 036101 (2012).

[8] B. S. Gottfried and K. J. Bell, Ind. Eng. Chem. Fundam. 5, 561 (1966).

[9] H. Linke, B. J. Alemán, L. D. Melling, M. J. Taormina, M. J. Francis, C. C. Dow-Hygelund, V. Narayanan, R. P. Taylor, and A. Stout, Phys. Rev. Lett. 96, 154502 (2006).

[10] D. Quéré and A. Ajdari, Nat. Mater. 5, 429 (2006).

[11] A. Snezhko, E. Ben Jacob, and I. S. Aranson, New J. Phys. 10, 043034 (2008).

[12] P. Brunet and J. H. Snoeijer, Eur. Phys. J. Special Top. 192, 207 (2011).
[13] G. Dupeux, M. Le Merrer, C. Clanet, and D. Quéré, Phys. Rev. Lett. 107, 114503 (2011).

[14] G. Lagubeau, M. Le Merrer, C. Clanet, and D. Quéré, Nat. Phys. 7, 395 (2011).

[15] F. Celestini, T. Frisch, and Y. Pomeau, Phys. Rev. Lett. 109, 034501 (2012).

[16] J. C. Burton, A. L. Sharpe, R. C. A. van der Veen, A. Franco, and S. R. Nagel, Phys. Rev. Lett. 109, 074301 (2012).

[17] I. U. Vakarelski, N. A. Patankar, J. O. Marston, D. Y. C. Chan, and S. T. Thoroddsen, Nature 489, 274 (2012).

[18] J. Bernardin and I. Mudawar, Trans. ASME 121, 894 (1999).

[19] J. H. Snoeijer, P. Brunet, and J. Eggers, Phys. Rev. E 79, 036307 (2009).

[20] Y. Pomeau, M. L. Berre, F. Celestini, and T. Frisch, arXiv:1208.2509v1.

[21] A. Onuki, Phys. Rev. Lett. 94, 054501 (2005).

[22] A. Onuki, Phys. Rev. E 75, 036304 (2007).

[23] X. Xu and T. Qian, J. Chem. Phys. 133, 204704 (2010).

[24] X. Xu, C. Liu, and T. Qian, Comm. Math. Sci. 10, 1027 (2012).

[25] X. Xu and T. Qian, Phys. Rev. E 85, 051601 (2012).

[26] X. Xu and T. Qian, Phys. Rev. E 85, 061603 (2012).

[27] D. M. Anderson and S. H. Davis, Phys. Fluids 7, 248 (1995). 
[28] H. Hu and R. G. Larson, Langmuir 21, 3972 (2005).

[29] V. S. Ajaev, J. Fluid Mech. 528, 279 (2005).

[30] N. Shahidzadeh-Bonn, S. Rafai, A. Azouni, and D. Bonn, J. Fluid Mech. 549, 307 (2006).

[31] L. Frastia, A. J. Archer, and U. Thiele, Phys. Rev. Lett. 106, 077801 (2011).

[32] T. Laurila, A. Carlson, M. Do-Quang, T. Ala-Nissila, and G. Amberg, Phys. Rev. E 85, 026320 (2012).

[33] R. Teshigawara and A. Onuki, Europhys. Lett. 84, 36003 (2008).

[34] R. Teshigawara and A. Onuki, Phys. Rev. E 82, 021603 (2010).

[35] X. Shan and H. Chen, Phys. Rev. E 47, 1815 (1993).

[36] A. Onuki, Phase Transition Dynamics (Cambridge University Press, Cambridge, 2002).

[37] D. M. Anderson, G. B. McFadden, and A. A. Wheeler, Annu. Rev. Fluid Mech. 30, 139 (1998).

[38] A. J. Briant, A. J. Wagner, and J. M. Yeomans, Phys. Rev. E 69, 031602 (2004).

[39] S. R. De Groot and P. Mazur, Non-Equilibrium Thermodynamics (Dover, New York, 1984).

[40] L. D. Landau and E. M. Lifshitz, Fluid Mechanics (ButterworthHeinemann, Oxford, 1987).

[41] J. Koplik, J. R. Banavar, and J. F. Willemsen, Phys. Rev. Lett. 60, 1282 (1988).

[42] P. A. Thompson and M. O. Robbins, Phys. Rev. Lett. 63, 766 (1989).

[43] T. Qian, X.-P. Wang, and P. Sheng, Phys. Rev. E 68, 016306 (2003).

[44] J.-L. Barrat and F. Chiaruttini, Mol. Phys. 101, 1605 (2003).
[45] J. B. Freund, Phys. Fluids 17, 022104 (2005).

[46] Z. Ge, D. G. Cahill, and P. V. Braun, Phys. Rev. Lett. 96, 186101 (2006).

[47] H. Kim, B. Truong, J. Buongiorno, and L.-W. Hu, Appl. Phys. Lett. 98, 083121 (2011).

[48] R. Teshigawara and A. Onuki, Phys. Rev. E 84, 041602 (2011).

[49] Y. Di and X.-P. Wang, J. Comput. Phys. 228, 1380 (2009).

[50] R. B. Bird, W. E. Stewart, and E. N. Lightfoot, Transport Phenomena (John Wiley, New York, 2002).

[51] J. W. Cahn, J. Chem. Phys. 66, 3667 (1977).

[52] A. Celani, A. Mazzino, P. Muratore-Ginanneschi, and L. Vozella, J. Fluid Mech. 622, 115 (2009).

[53] R. Borcia, I. D. Borcia, and M. Bestehorn, Eur. Phys. J. Special Top. 166, 127 (2009).

[54] P. Yue, C. Zhow, and J. J. Feng, J. Fluid Mech. 645, 279 (2010).

[55] W. D. Ristenpart, P. G. Kim, C. Domingues, J. Wan, and H. A. Stone, Phys. Rev. Lett. 99, 234502 (2007).

[56] A. Würger, Phys. Rev. Lett. 107, 164502 (2011).

[57] E. S. Godlesk and M. J. Bell, Proc. Intern. Heat Transfer Conf. 4, 118 (1966).

[58] U. Thiele, S. Madruga, and L. Frastia, Phys. Fluids 19, 122106 (2007).

[59] A. Onuki, Phys. Rev. E 79, 046311 (2009).

[60] U. Grigull, J. Straub, and P. Schiebener, Steam Tables in SI-Units (Springer-Verlag, Berlin, 1990).

[61] V. Sychev and T. B. Selover, Thermodynamic Properties of Nitrogen (Hemisphere, Washington, D.C., 1987). 